\title{
Benzene degradation by Ralstonia pickettii PKO1 in the presence of the alternative substrate succinate
}

\author{
Margarete Bucheli-Witschel · Tina Hafner · \\ Irene Rüegg • Thomas Egli
}

Received: 28 March 2008/Accepted: 27 October 2008/Published online: 28 November 2008

(C) Springer Science+Business Media B.V. 2008

\begin{abstract}
The regulation of benzene degradation by Ralstonia pickettii PKO1 in the presence of the alternative substrate succinate was investigated in batch and continuous culture. In batch culture, $R$. pickettii PKO1 achieved a maximum specific growth rate with benzene of $0.18 \mathrm{~h}^{-1}$, while succinate allowed much faster growth $\left(\mu_{\max }=0.5 \mathrm{~h}^{-1}\right)$. Under carbon excess conditions succinate repressed benzene consumption resulting in diauxic growth whereas under carbon-limited conditions in the chemostat both substrates were used simultaneously. Moreover, the effect of succinate on the adaptation towards growth with benzene was investigated in carbon-limited continuous culture at a dilution rate of $0.1 \mathrm{~h}^{-1}$ by changing the inflowing carbon substrate from succinate to different mixtures of benzene and succinate. The adaptation process towards utilisation of benzene was rather complex. Three to seven hours after the medium shift biomass production from benzene started. Higher proportions of succinate in the mixture had a positive effect on both the onset of biomass production and on the time required for induction of benzene utilisation. Strikingly, after the
\end{abstract}

M. Bucheli Witschel · T. Hafner · I. Rüegg · T. Egli ( $\varangle)$

Department of Environmental Microbiology, Eawag,

Swiss Federal Institute of Aquatic Science

and Technology, Überlandstrasse 133,

P. O. Box 611, 8600 Dübendorf, Switzerland

e mail: egli@eawag.ch initial increase in biomass and benzene-catabolising activities, the culture collapsed regularly and washout of biomass was observed. After a transient phase of low biomass concentrations growth on benzene resumed so that finally rather stable and high biomass concentrations were reached. The decrease in biomass and degradative activities cannot be explained so far, but the possibilities of either intoxication of the cells by benzene itself, or of inhibition by degradation intermediates were ruled out.

Keywords Benzene - Continuous culture . Adaptation process - Ralstonia pickettii PKO1 . Succinate
Abbreviations
BTEX Benzene, toluene, ethylbenzene and xylenes
OD Optical density
DOC Dissolved organic carbon
NTA Nitrilotriacetate
3-ppa 3-Phenylpropionic acid

\section{Introduction}

Contamination of groundwater with petroleum products such as the water-soluble monoaromatic compounds benzene, toluene, ethylbenzene and xylenes (BTEX) is a major environmental problem (Yerushalmi et al. 1999). As a promising approach for the clean-up of polluted aquifers, microbial degradation of BTEX 
compounds is often suggested (Gulensoy and Alvarez 1999). Consequently, a wide variety of bacterial strains able to degrade BTEX compounds has been isolated and considerable effort was put in elucidating the genetic and biochemical phenomena associated with aerobic, microaerophilic and anaerobic BTEX degradation (Smith 1994; Jindrova et al. 2002; Olsen et al. 1995; Spormann and Widdel 2000; Lovley 2000). At the molecular level, the regulation of genes involved in BTEX catabolism has received much attention and several regulators (e.g. XylR, TbuT, TodST) have been characterised thoroughly (Tropel and van der Meer 2004; Shingler 2003). Nevertheless, there is a paucity of information concerning the physiological aspects of the regulation of BTEX mineralization. The effects of several factors on BTEX catabolism and its regulation, e.g. the presence of alternative, easily degradable substrates besides the pollutant(s), growth under carbon/energy-limited conditions, or limited availability of terminal electron acceptors, are only poorly understood. In natural aquatic systems, however, being mainly carbon-limited (Morita 1993), bacteria probably use and grow with xenobiotic compounds such as BTEX and easily degradable carbon sources at the same time. In addition, bacteria are not only confronted with mixtures of carbon substrates but also with an ever changing environment including changes in substrate availability and spectrum, resulting for example from groundwater flow and heterogeneous source distribution (see, e.g. Vroblesky and Chapelle 1994).

To study the influence of an alternative substrate on the degradation of aromatic compounds, Ralstonia pickettii $\mathrm{PKO} 1$ was chosen as model organism. This bacterium is able to grow with benzene, toluene, phenol and $m$-cresol as sole sources of carbon and energy under both aerobic and oxygen-limited, denitrifying conditions (Leahy and Olsen 1997; Bruins et al. 2000). The initial step of benzene metabolism involves the hydroxylation of benzene to phenol, which is then further hydroxylated to catechol, prior to ring cleavage by a meta-fission dioxygenase. The genes encoding the enzymes for toluene and benzene utilisation ( $t b u$ genes) are organised in four operons: the tbuAIUBVA2C and tbuT operon encoding the initial toluene-3-monooxygenase and the regulatory protein TbuT, the $t b u D$ operon encoding phenol/ cresol hydroxylase, the catechol meta cleavage operon, tbuWEFGKIHJ, and finally the $t b u X$ locus, the gene product of which might be involved in facilitating toluene/benzene uptake. All operons are under the control of the transcriptional activator TbuT (Olsen et al. 1997; Kahng et al. 2000). A broad effector specificity was shown for TbuT with toluene, ethylbenzene, benzene and chlorobenzene being the strongest inducers (Park et al. 2003). Furthermore, it has recently been shown that the rate of toluene degradation by PKO1 decreased exponentially during periods of carbon starvation. This deactivation probably resulted from carbon starvation itself and not from the absence of an inducer of the $t b u$ pathway (Johnson et al. 2006).

Here we investigated the dynamics of benzene degradation by strain PKO1 in the presence of the alternative substrate succinate under both batch and continuous culture conditions. In the latter case, the main focus was not put on steady-state but rather on transient conditions concerning the substrate supply to study the adaptation process towards utilisation of benzene and the role of the alternative substrate in it.

\section{Material and methods}

Microbial strains and cultivation

Ralstonia pickettii PKO1 was used for all experiments. The bacteria were cultured at $30^{\circ} \mathrm{C}$ in Evans mineral medium (Evans et al. 1970) diluted to $25 \%$ of its original strength. The medium was modified by supplying $162 \mathrm{mg} \mathrm{l}^{-1} \mathrm{CaCl}_{2} \cdot 2 \mathrm{H}_{2} \mathrm{O}$, by replacing citric acid with EDTA $(0.08 \mathrm{mM})$, and by adding $1 \mathrm{ml} \mathrm{l}^{-1}$ vitamin solution (Egli et al. 1988). For batch cultivation the $\mathrm{pH}$ was adjusted to 7.0 using potassium phosphate buffer $(50 \mathrm{mM})$ while for chemostat cultivation no buffer was added but the medium was acidified with $\mathrm{H}_{3} \mathrm{PO}_{4}(8.7 \mathrm{mM})$.

From stock cultures material was plated on $10 \%$ tryptic soy agar plates and incubated at $30^{\circ} \mathrm{C}$ until single colonies developed. For pre-cultures one colony was inoculated into $50 \mathrm{ml}$ of mineral medium. As carbon sources, $5 \mathrm{mM}$ succinate was added directly to the medium, whereas benzene and toluene were supplied via the vapour phase $(0.2 \mathrm{ml}$ each, dissolved in $1.8 \mathrm{ml} n$-hexadecane, placed in open $10 \mathrm{ml}$ pyrex tubes, and added to the closed cultivation bottles so that diffusion via the air phase into the medium 
occurred). The pre-cultures with the aromatic solvents were additionally supplemented with $0.1 \mathrm{mg} \mathrm{l}^{-1}$ casamino acids to support growth.

\section{Batch cultures}

To determine the maximum specific growth rates of R. pickettii $\mathrm{PKO} 1$ with benzene $(2 \mathrm{mM})$, toluene $(2 \mathrm{mM})$, mixtures of benzene/succinate (1 and $0.5 \mathrm{mM}$, respectively), and mixtures of benzene/ toluene ( $1 \mathrm{mM}$, each), cells were cultivated in $250 \mathrm{ml}$ mineral medium in sealed $500 \mathrm{ml}$ flasks at $30^{\circ} \mathrm{C}$ under batch conditions. Benzene and toluene were directly added to the mineral medium and the medium was stirred for $12 \mathrm{~h}$ to equilibrate the system without micro-organisms. The maximum specific growth rate with succinate $(1.25 \mathrm{mM})$ was analysed in a bioreactor with 21 working volume (MBR, Wetzikon, Switzerland) at $30^{\circ} \mathrm{C}$. The inocula for batch growth experiments were taken from the second pre-culture grown with the respective substrate. For growth experiments with substrate mixtures the bacteria were pre-cultured twice with succinate.

\section{Chemostat cultures}

For continuous cultivation the dilution rate $(D)$ was kept at $0.1 \mathrm{~h}^{-1}$. The working volume of the bioreactor (MBR) was 21 . The $\mathrm{pH}$ in the cultivation liquid was automatically regulated at 7.0 by adding a $\mathrm{KOH} /$ $\mathrm{NaOH}$ mixture $(0.5 \mathrm{M}$, each). The temperature was maintained at $30^{\circ} \mathrm{C}$, and the cultivation broth was stirred with $800 \mathrm{rpm}$. Moreover, the reactor was aerated with air at a flow rate of $0.51 \mathrm{~min}^{-1}$ to achieve an oxygen saturation of $80100 \%$.

In all medium shift experiments, the bacteria were initially cultivated with $1.25 \mathrm{mM}$ succinate as sole source of carbon and energy until steady-state conditions were achieved. Then the composition of the inflowing medium was shifted to either benzene only, or to various mixtures of benzene and succinate as indicated in the results section. Benzene was supplied to the inflowing medium by a syringe pump (Indulab AG, Gams, Switzerland) to obtain a concentration of $2 \mathrm{mM}$ in the feed. The syringes used for adding benzene to the medium and taking benzene samples from the bioreactor were gastight (Hamilton, Nevada, USA). To prevent loss of benzene by adsorption or diffusion, all tubes, which were in contact with benzene, were made of Teflon or Viton rubber (Milian, Geneva, Switzerland).

A wash-in curve for benzene was determined assuming that benzene is not degraded and using a Henry's law-type distribution coefficient $H=0.22$ (Stumm and Morgan 1981) to consider the equilibrium of benzene between water and gas phase. According to these calculations the final concentration of benzene in the cultivation liquid is reached within the first $12 \mathrm{~h}$ and amounts to $60 \mu \mathrm{M}$. This value was verified in a sterile control reactor run at a dilution rate $D=0.1 \mathrm{~h}^{-1}$ and supplied with a mineral medium containing $2 \mathrm{mM}$ benzene. Benzene concentrations in the reactor liquid and benzene loss via the exhaust air were determined under steadystate conditions. In the reactor liquid, solvent concentrations varied between 40 and $90 \mu \mathrm{M}$ and at the same time, $294.5 \pm 64 \mu \mathrm{mol}$ benzene $\mathrm{h}^{-1}$ were lost over the headspace corresponding to about $75 \%$ of the incoming benzene.

\section{Biomass determination}

Growth of $R$. pickettii PKO1 was followed by measuring the optical density (OD) of the liquid culture in a $1 \mathrm{~cm}$ cuvette at $546 \mathrm{~nm}$ with an Uvikon 860 spectrophotometer (Kontron Instruments, Watford, UK).

Substrate-induced oxygen uptake rates in whole cells

Culture suspension was collected from the bioreactor, and cells were harvested by centrifugation at $20000 \mathrm{~g}$ and $4{ }^{\circ} \mathrm{C}$ for $10 \mathrm{~min}$. The pellet was resuspended in $50 \mathrm{mM}$ potassium-phosphate-buffer $(\mathrm{pH} \mathrm{7.0)}$ to obtain an $\mathrm{OD}_{546}$ of at least 0.08 . Substrate-stimulated oxygen uptake was recorded at $30^{\circ} \mathrm{C}$ in a Clark type oxygen cell (Rank Brothers Ltd, Bottisham, England). After determination of the endogenous oxygen consumption rate of $1.0 \mathrm{ml}$ cell suspension in the absence of substrate, $10 \mu \mathrm{l}$ of a substrate stock solution $(0.25 \mathrm{mM}$ benzene dissolved in methanol, or phenol, catechol, or succinate, $10 \mathrm{mM}$, each) were added. The substrate-induced oxygen uptake rate was calculated using an oxygen solubility in water at $30^{\circ} \mathrm{C}$ of $7.53 \mathrm{mg} \mathrm{O}_{2} 1^{-1}$ (Sigg and Stumm 1996). 
Analytical methods

For all analysis (except for the determination of 2-hydroxymuconate semialdehyde) calibration curves were prepared by using known concentrations of the respective compound dissolved in mineral medium without carbon source. In case of dissolved organic carbon (DOC) determinations potassium hydrogenphthalate was used as standard.

\section{Benzene and toluene}

The analytical procedure for the separation of benzene and toluene components was based on solid phase micro extraction (SPME), subsequent gas chromatography (GC) and flame ionisation detection (FID). The solid phase for extraction was integrated in the needle of the GC apparatus and consisted of polydimethylsiloxane (Supelco, Bellefonte, USA). For GC, a $30 \mathrm{~m}$ capillary column SolGel-Wax ${ }^{\mathrm{TM}}$ (Infochrom AG, Zug, Switzerland) was chosen and as a carrier gas helium was used. Cultivation liquid $(1.6 \mathrm{ml})$ was acidified with a drop of $32 \% \mathrm{HCl}$ and then subjected to SPME GC. During a $10 \mathrm{~min}$ absorption period at $25^{\circ} \mathrm{C}$, benzene was extracted, to be subsequently desorbed within $3 \mathrm{~min}$. The injector temperature was set at $250^{\circ} \mathrm{C}$. Column temperature was programmed to increase in a first step from an initial value of $4595^{\circ} \mathrm{C}$ at a rate of $10^{\circ} \mathrm{C} \mathrm{m^{-1 }}$ and in a second step from 95 to $200^{\circ} \mathrm{C}$ at a rate of $20^{\circ} \mathrm{C} \mathrm{min}^{-1}$. The FID detector was operated at $300^{\circ} \mathrm{C}$. The detection limit for benzene and toluene was below $1 \mu \mathrm{M}$.

Benzene loss over the headspace of the bioreactor was determined by tracking the exhaust air in two wash bottles filled with ethanol, which were connected in series. In a time period of $2 \mathrm{~h}$ both wash bottles were sampled every $15 \mathrm{~min}$ and benzene concentrations in ethanol were measured by means of high performance liquid chromatography (HPLC, Gynkotec, Munich, Germany), using a reverse phase LiChroCart C18 separation column (Merck, NY, USA) and a variable wavelength detector (UVD 340U, Dionex, Olten, Switzerland) set at $254 \mathrm{~nm}$. Acetonitrile:water (70:30) at a flow rate $1 \mathrm{ml} \mathrm{min}{ }^{-1}$ served as eluent.

Prior to the following analyses, cells were removed from the cultivation liquid by filtration through a $0.2 \mu \mathrm{m}$ pore polycarbonate membrane filter (Millipore, Volketswil, Switzerland):
Phenol and catechol

The pathway intermediates phenol and catechol were analysed by HPLC (Gynkotec). A volume of $10 \mu \mathrm{l}$ of the acidified, cell-free sample was injected into the apparatus equipped with a reverse phase LiChroCart C18 separation column (Merck) and a variable wavelength detector (UVD 340U, Dionex) set at $207 \mathrm{~nm}$. The compounds were eluted with a $\mathrm{NaH}_{2} \mathrm{PO}_{4}(50 \mathrm{mM}, \mathrm{pH} 3.0)$ gradient at a flow rate of $0.7 \mathrm{ml} \mathrm{min}^{-1}$. After 26 min elution with solution A (30\% methanol, $70 \% \quad \mathrm{NaH}_{2} \mathrm{PO}_{4}$ ), the eluent was changed within 2 min to $25 \%$ solution $\mathrm{A}$ and $75 \%$ solution B (90\% methanol, $\left.10 \% \quad \mathrm{NaH}_{2} \mathrm{PO}_{4}\right)$. The detection limits for phenol and catechol were about 1 and $10 \mu \mathrm{M}$, respectively.

\section{Succinate}

For succinate determination ion exclusion chromatography (Dionex) was used. The column for separation of $50 \mu \mathrm{l}$ of an acidified sample was an Ion-Pac ICE-AS6 (Dionex). $0.4 \mathrm{mM}$ heptafluorobutyric acid served as an eluent and was delivered to the system at a flow rate of $1 \mathrm{ml} \mathrm{m^{-1 }}$. Upstream of the conductivity detector (ECD50, Dionex) an anion micro membrane suppressor (AMMS-ICE, Dionex) was installed, which was regenerated by $5 \mathrm{mM}$ tetrabutylammonium-hydroxide at a flow rate of $5 \mathrm{ml} \mathrm{min}{ }^{-1}$. The detection limit for succinate determination was below $10 \mu \mathrm{M}$.

\section{Dissolved organic carbon (DOC)}

The filtrate $(5 \mathrm{ml})$ was acidified with $32 \% \mathrm{HCl}, \mathrm{CO}_{2}$ was stripped with nitrogen gas and DOC was measured with the total organic carbon analyser TOCOR 2 (Maihak AG, Hamburg, Germany). In order to check whether the carbon content of semivolatile substances (e.g. phenol) was exactly determined with this method, phenol solutions of different concentrations were measured. The results indicated that there was a very good agreement between the theoretical and the experimental data.

\section{2-Hydroxymuconate-semialdehyde}

The meta-ring fission product of catechol was spectrophotometrically determined with an Uvikon 
860 spectrophotometer at $\lambda=375 \mathrm{~nm}$. To calculate the concentration an extinction coefficient of $\varepsilon=36 \mathrm{mM}^{-1} \mathrm{~cm}^{-1}$ (Heiss et al. 1995) was used.

\section{Chemicals and reagents}

Catechol was purchased from Sigma (St Louis, USA), casamino acids and tryptic soy agar from Biolife (Milan, Italy). All other chemicals were of analytical grade and obtained from Fluka (Buchs, Switzerland).

\section{Results and discussion}

Growth of $R$. pickettii $\mathrm{PKO} 1$ in batch culture

Despite the wealth of information available on the biochemistry and genetics of benzene/toluene catabolism of this strain (Kukor and Olsen 1990, 1991, 1992, 1996; Olsen et al. 1994, 1997; Byrne et al. 1995; Kahng et al. 2000; Tao et al. 2004), little has so far been reported on its growth behaviour. Therefore, growth and degradation properties of $R$. pickettii PKO1 were investigated in a first step under carbon excess conditions. Batch experiments with single substrates (succinate, benzene and toluene) and mixtures of substrates (succinate and benzene, benzene and toluene) were performed at $30^{\circ} \mathrm{C}$.

With succinate as sole source of carbon and energy a maximum specific growth rate $\left(\mu_{\max }\right)$ of $0.5 \pm 0.09 \mathrm{~h}^{-1}$ was observed. Growth with the tested monoaromatic compounds was 23 times slower. With either benzene or toluene $\mu_{\max }$ was $0.18 \pm 0.05 \mathrm{~h}^{-1}$ and $0.17 \pm 0.04 \mathrm{~h}^{-1}$, respectively. Based on the measurement of oxygen uptake in a respirometer, Alagappan and Cowan (2003) derived $\mu_{\max }$ values of strain PKO1 during growth with benzene and toluene at $20^{\circ} \mathrm{C}$. As in our experiments, the rates were similar to each other, but they were 1.72 times higher than those we recorded. In contrast, Massol-Deya et al. (1997) reported a $\mu_{\max }$ with toluene of $0.16 \mathrm{~h}^{-1} \quad\left(T=25^{\circ} \mathrm{C}\right)$, which corresponds well with the value obtained in this study.

When $R$. pickettii PKO1 (pre-grown with succinate) was cultivated with a mixture of benzene and toluene at an initial concentration of about $1 \mathrm{mM}$ each, it degraded both substrates simultaneously. The $\mu_{\max }$ supported during the exponential growth phase was $0.25 \pm 0 \mathrm{~h}^{-1}$, which was distinctly higher than the growth rates $R$. pickettii PKO1 achieved during growth with these substrates when supplied on their own. For several other microorganisms such a stimulation of $\mu_{\max }$ was also observed in case of simultaneous utilisation of mixtures of carbon substrates when compared to growth with the individual carbon sources (Egli 1995).

When transferring strain PKO1 from succinate to a mixture of succinate and benzene (1 mM each) a diauxic growth pattern was observed, with succinate being the preferred substrate (Fig. 1). After growth on succinate the cells entered a lag phase of $34 \mathrm{~h}$ during which no biomass was produced. However, benzene degradation began during this phase and it was accompanied by the transient accumulation of phenol up to $\sim 50 \mu \mathrm{M}$. Phenol excretion was not stoichiometric to benzene utilisation and amounted to only $1020 \%$ of the benzene degraded indicating that most of the phenol produced was further catabolised. Catechol concentrations were always below the detection limit of $10 \mu \mathrm{M}$. Finally, at no point accumulation of the yellow catechol-fission product, 2-hydroxymuconate semialdehyde, was detected. The delayed biomass production from benzene might be explained by the fact that intermediates of benzene degradation, in particular phenol, rather than the substrate itself supported the growth of cells as described for BTX degradation by Pseudomonas putida F1 (Yu et al. 2001). During both growth phases the bacteria grew exponentially. However,

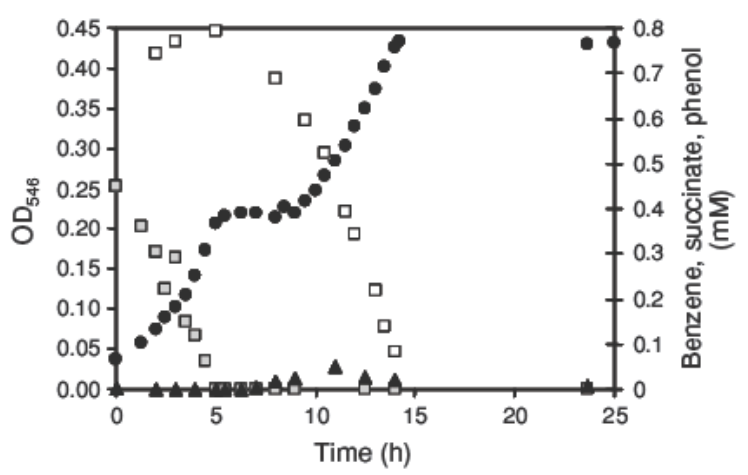

Fig. 1 Batch growth of $R$. pickettii PKO1 with a mixture of succinate and benzene as sole source of carbon and energy. Growth was monitored as $\mathrm{OD}_{546}(-)$ and the concentrations of the substrates succinate $(\square)$ and benzene $(\square)$ were measured. Moreover, phenol $(\boldsymbol{\Delta})$, an intermediate of benzene degradation, was detected 
during growth with succinate $\left(\mu_{\max }=0.32 \pm 0.04\right)$ as well as during growth with benzene ( $\mu_{\max }=$ $0.1 \pm 0.04) \mu_{\max }$ was diminished by a factor of 1.52 when compared to the specific growth rates found during cultivation with the individual substrates.

Based on results from batch cultivations, a dilution rate of $D=0.1 \mathrm{~h}^{-1}$ was chosen for the subsequent continuous culture experiments because it was lower than the maximum specific growth rates of strain PKO1 with all substrates of interest.

Transitions from succinate to mixtures of benzene plus succinate or to benzene only

The influence of the availability of a second, easily degradable substrate (succinate) on the process of inducing the ability to degrade benzene in $R$. pickettii PKO1 was investigated in carbon-limited continuous culture by subjecting the cells to shifts from succinate to either different mixtures of benzene plus succinate, or to benzene only. Each transition experiment was started from an established steady-state on a medium containing $1.25 \mathrm{mM}$ succinate only, which was then replaced with a medium containing $2.0 \mathrm{mM}$ benzene supplemented with $1.25,0.35,0.125 \mathrm{mM}$ or containing benzene $(2 \mathrm{mM})$ only. Consequently, the media contained $71,90,96$ or $100 \%$ carbon stemming from benzene. To test for reproducibility, the shift from $1.25 \mathrm{mM}$ succinate to $1.25 \mathrm{mM}$ succinate plus $2 \mathrm{mM}$ benzene was performed in two independent chemostat experiments. Both experiments showed the same overall behaviour of the cultures as described below.

\section{The first hours after the medium shift: induction of benzene degradation}

In all transition experiments the growth behaviour of R. pickettii PKO1 was similar (Fig. 2a). When the succinate concentration in the medium feed was kept constant, biomass formation from benzene started about $3 \mathrm{~h}$ after the transition. When the succinate concentration in the medium feed was reduced the biomass decreased according to the theoretical washout curve, assuming that the cells grew only with succinate. After about $5 \mathrm{~h}$ the $\mathrm{OD}_{546}$ began to increase again. Omitting succinate from the medium resulted in wash-out of biomass during $7 \mathrm{~h}$ and then a plateau was attained with OD values around 0.075 . Thus, higher portions of carbon stemming from
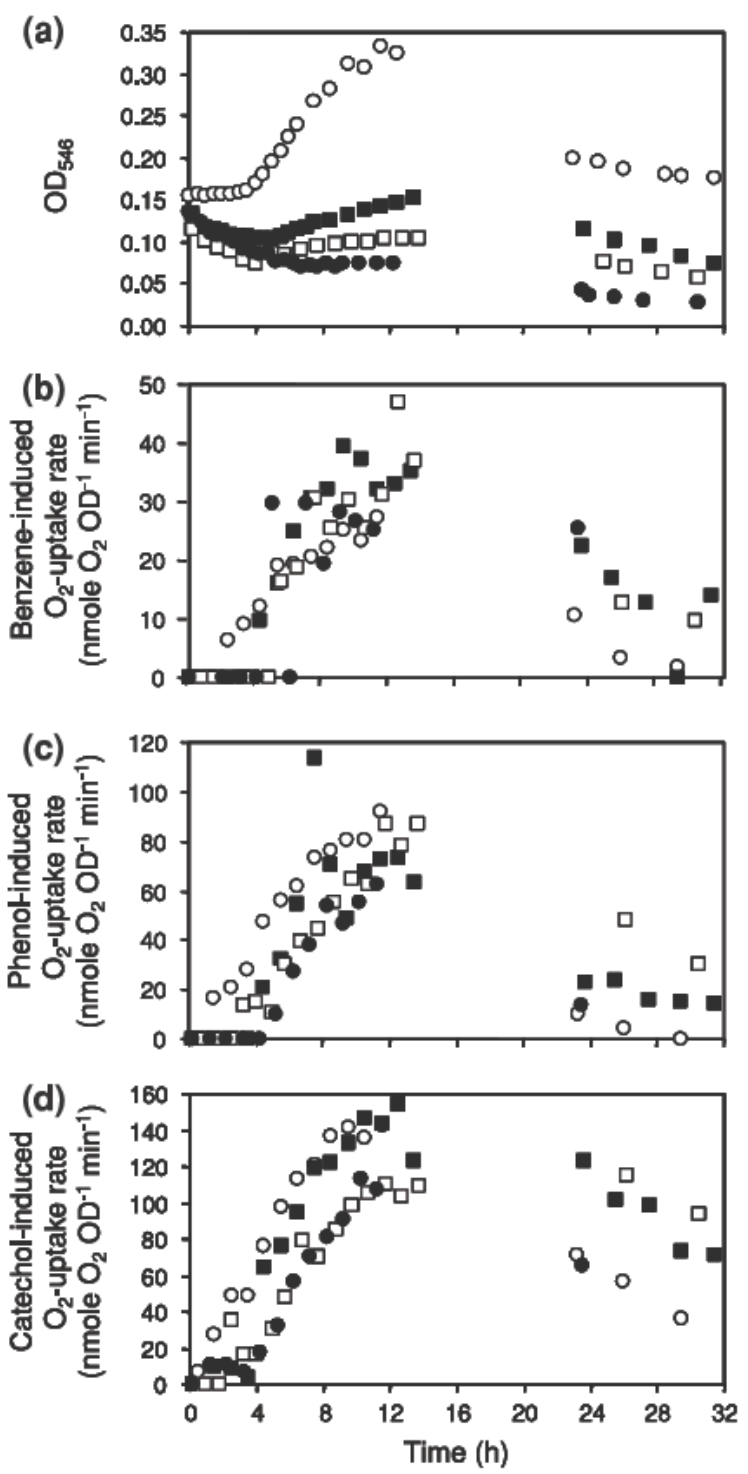

Fig. 2 Transient behaviour of $R$. pickettii PKO1 grown in carbon limited continuous culture $\left(\begin{array}{ll}D & 0.1 \mathrm{~h}^{-1}\end{array}\right)$ after switch ing the feed from a medium containing succinate $(1.25 \mathrm{mM})$ as sole source of carbon and energy to a medium containing a mixture of $2 \mathrm{mM}$ benzene and $1.25 \mathrm{mM}(0), 0.35 \mathrm{mM}(\square)$, $0.125 \mathrm{mM}(\square)$ succinate, or no succinate $(O)$. Time course of the biomass concentration monitored as $\mathrm{OD}_{546}$ (a) and of the benzene (b), phenol (c) and catechol induced (d) oxygen uptake rates in whole cells in the first $36 \mathrm{~h}$ after the switch

succinate in the medium after the switch slightly reduced the time until biomass formation from benzene started. The rise in biomass was accompanied by increasing benzene-, phenol- and catecholinduced oxygen uptake rates (Fig. $2 b$ d). When the succinate concentration before and after the shift was 
kept constant, enzymatic activities were induced $23 \mathrm{~h}$ earlier than in all shifts where succinate concentrations were reduced or where succinate was omitted. Moreover, in all transition experiments, catechol-stimulated oxygen uptake rate was up-regulated first, followed by phenol- and benzene-induced oxygen uptake rates. Interestingly, when the succinate concentration in the feed was reduced, the catechol-triggered oxygen uptake rates were slightly induced over the background level already from about $1 \mathrm{~h}$ on after the shift; however, a marked rise of activity started only about $45 \mathrm{~h}$ after the medium change.

Compared with the situation found for other pollutant-degrading bacteria, the supply of an alternative substrate only slightly supported the dynamics of adaptation to growth with a monoaromatic compound in $R$. pickettii PKO1. For instance, a more dramatic positive effect of an alternative substrate on the adaptation process was observed in the nitrilotriacetate (NTA)-degrader Chelatobacter heintzii (Bally and Egli 1996) and in the benzene/toluene-degrading strain Pseudomonas putida F1 (Rüegg et al. 2007). In C. heintzii the lag phase until expression of the NTAconverting enzyme started was markedly shortened to $<9 \mathrm{~h}$ when the cells were subjected to shifts in the medium supply from glucose to mixtures of glucose and NTA; whereas for the transition from glucose to NTA alone the delay until induction started lasted about $25 \mathrm{~h}$. Also for strain F1, higher portions of succinate in the medium reduced the time period considerably until biomass production occurred. In contrast, enzyme induction in this strain was merely dependent on the presence or absence of succinate. As long as succinate was present enzyme activities involved in benzene/toluene catabolism began to rise around $12 \mathrm{~h}$ after the shift whereas in the absence of succinate a lag time of more than $10 \mathrm{~h}$ was observed. Although it might be premature to deduce a general rule the three systems analysed suggest that under carbon-limited conditions the presence of an alternative substrate does not slow down the adaptation process towards pollutant degradation but rather accelerates it. This supporting effect is possibly due to the alternative substrate supplying energy and building blocks for the de novo synthesis of enzymes (Egli 2002). Moreover, the extent of upregulation of genes, in particular, of stress genes, in response to a xenobiotic compound might be reduced when an alternative substrate is present after the transitions, as has been shown for P. putida (del Castillo and Ramos 2007), thereby reducing the energetic expense for the adaptation process.

Interestingly, the sequence of the start of induction of enzyme activities, i.e. catechol-degrading activity appeared before phenol-transforming activity, and the latter again before or equal with benzene-degrading activity, was opposed to the succession of the enzymatic activities in the catabolic pathway. Similarly, in $P$. putida F1 benzene/toluene-degrading activity lagged always $12 \mathrm{~h}$ behind the onset of the enzyme activity for the degradation of the intermediate catechol (Finette and Gibson 1988; Rüegg et al. 2007). Possibly, the early induction of catechol-transforming activities in $R$. pickettii $\mathrm{PKO} 1$ and $P$. putida F1 helps the cells to prevent intoxication by accumulation of pathway intermediates such as catechol and phenol (Munoz et al. 2007). In R. pickettii, the enzymes catalysing the investigated transformations, i.e. benzene/toluene monooxygenase, phenol/cresol hydroxylase, and catechol-2,3-dioxygenase, are encoded by genes located on three different operons of the $t b u$ regulon, all of which are under the transcriptional control of the activator TbuT (Olsen et al. 1997). Thus the succession of induction is compatible with the genetic organisation. However, there is evidence that the promoter of the benzene/ toluene monooxygenase operon drives the expression of the gene for the regulator protein TbuT by readthrough transcription (Byrne and Olsen 1996). This information makes it difficult to conceive why the enzymes encoded by the $t b u$ meta-pathway operon were significantly earlier expressed than the enzyme catalysing the initial attack on benzene/toluene.

Long-term behaviour of the cultures after the medium shift: unstable growth with benzene

Strikingly, the first formation of biomass from benzene did not lead to a stable steady-state situation but biomass began to decrease again $1524 \mathrm{~h}$ after the shift; this pattern was independent from the succinate concentration in the medium feed (Fig. 2a). Concomitant with the development of the biomass concentration in the bioreactor also the benzene-, phenol- and catechol-stimulated oxygen uptake rates reached a temporary maximum and decreased thereafter (Fig. 2b d). Interestingly, a similar behaviour was 
also observed for medium shifts from succinate to various mixtures of succinate and benzene in a carbonlimited continuous culture of $P$. putida F1 (Rüegg et al. 2007). So far, no explanation for this surprising pattern of the adaptation dynamics of strain $\mathrm{F} 1$ has been found.

It is possible that the observed decrease in biomass resulted from a loss of benzene-degradative activity in a subpopulation of the culture, as it has been found in other hydrocarbon-degrading bacteria during subculturing even if the hydrocarbon was provided as only source of carbon and energy (Leddy et al. 1995). However, although not experimentally tested a loss of benzene-degrading functions during any time of the precultivation in batch and during chemostat cultivation seems to be rather unlikely because such phenomena are generally described for plasmidencoded pathways (e.g. Duetz and van Andel 1991; Leddy et al. 1995). For that reason, a strain was chosen for this study in which all hydrocarbondegrading genes are located on the chromosome to circumvent interference by plasmid instability. Moreover, even when assuming the loss of benzenecatabolising functions in a subpopulation, it is not conceivable what factor triggered this loss after the first period of biomass formation as a result of benzene utilisation.

To investigate whether a stable situation would finally be reached characterised by low biomass concentrations or whether growth would resume as found for $P$. putida F1, two chemostats were run for up to $400500 \mathrm{~h}$ after the switch in the inflowing medium from succinate only to either $2 \mathrm{mM}$ benzene plus $1.25 \mathrm{mM}$ succinate or to $2 \mathrm{mM}$ benzene only. In the first case, $\mathrm{OD}_{546}$ values were as low as during growth with succinate only in the time period between 50 and $80 \mathrm{~h}$ after the medium change (Fig. 3). Then biomass concentration started to increase again, however, was fluctuating considerably during the next $200 \mathrm{~h}$ until the culture became more stable at $\mathrm{OD}_{546}$ values of 0.4 and 0.45 . Assuming batch growth yields with benzene, this final biomass concentration suggested the utilisation of about $1 \mathrm{mM}$ benzene in addition to the complete consumption of succinate. In the whole course of the experiment the patterns for aromatic compound-transforming activities followed the biomass development.
Fig. 3 Transient behaviour of $R$. pickettii $\mathrm{PKO} 1$ grown in carbon limited continuous culture (D $0.1 \mathrm{~h}^{-1}$ ) after switching the feed from a medium containing succinate $(1.25 \mathrm{mM})$ only to one containing a mixture of $1.25 \mathrm{mM}$ succinate and $2 \mathrm{mM}$ benzene. a Time course of the biomass concentration monitored as $\mathrm{OD}_{546}(\mathrm{O})$ and benzene induced oxygen uptake rate in whole cells $(\diamond)$. b Time course of the benzene ( $\square$ ) and phenol ( $\mathbf{\Delta})$ concentrations in the cultivation liquid
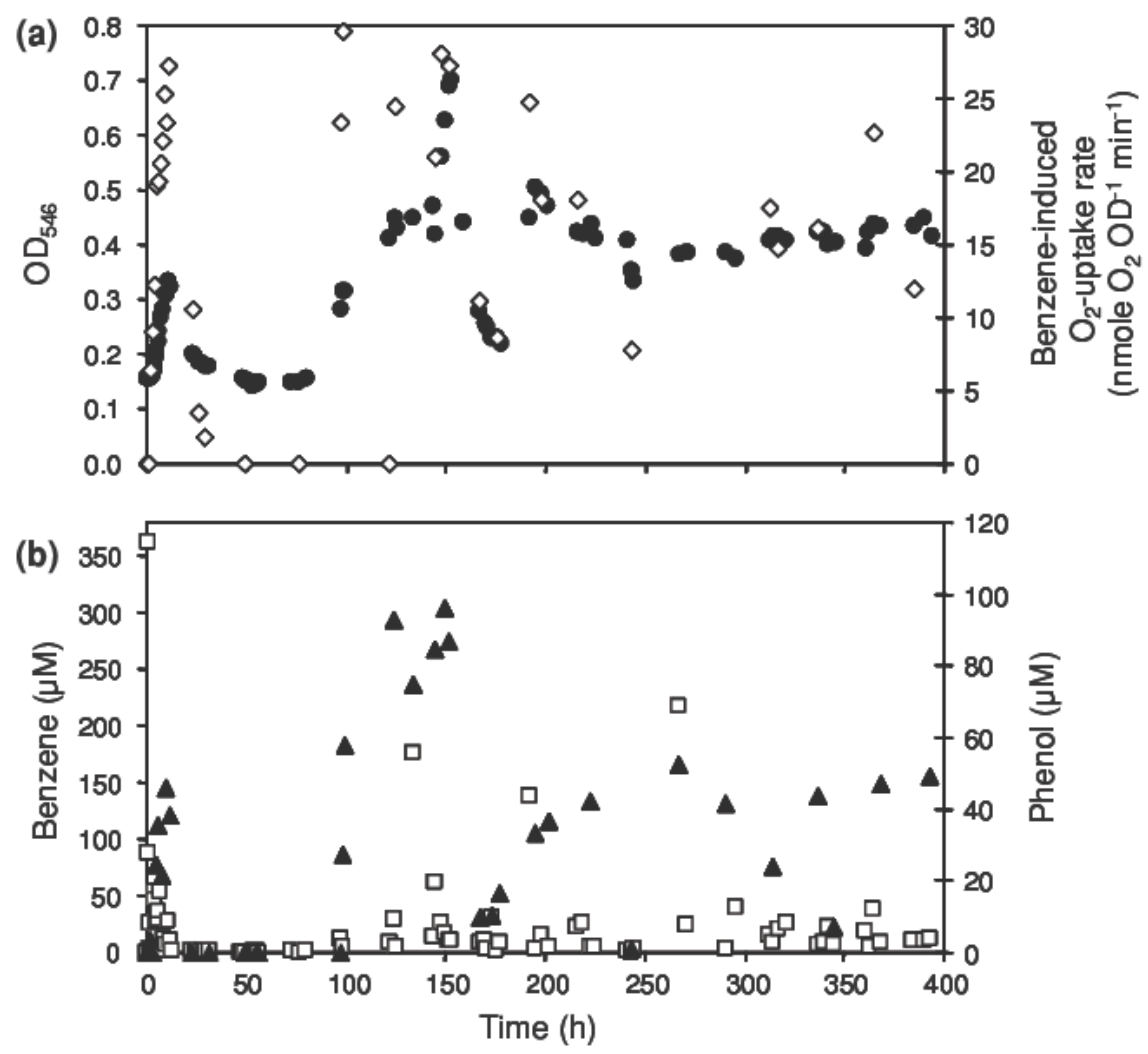
Fig. 4 Transient behaviour of $R$. pickettii $\mathrm{PKO} 1$ grown in carbon limited continuous culture (D $0.1 \mathrm{~h}^{-1}$ ) after switching the feed from a medium containing succinate $(1.25 \mathrm{mM})$ as sole source of carbon and energy to one containing $2 \mathrm{mM}$ benzene only. a Time course of the biomass concentration monitored as $\mathrm{OD}_{546}(\mathrm{O})$ and benzene induced oxygen uptake rate in whole cells $(\diamond)$. b Time course of the benzene ( $\square$ ) and phenol ( $\mathbf{\Delta})$ concentrations in the cultivation liquid
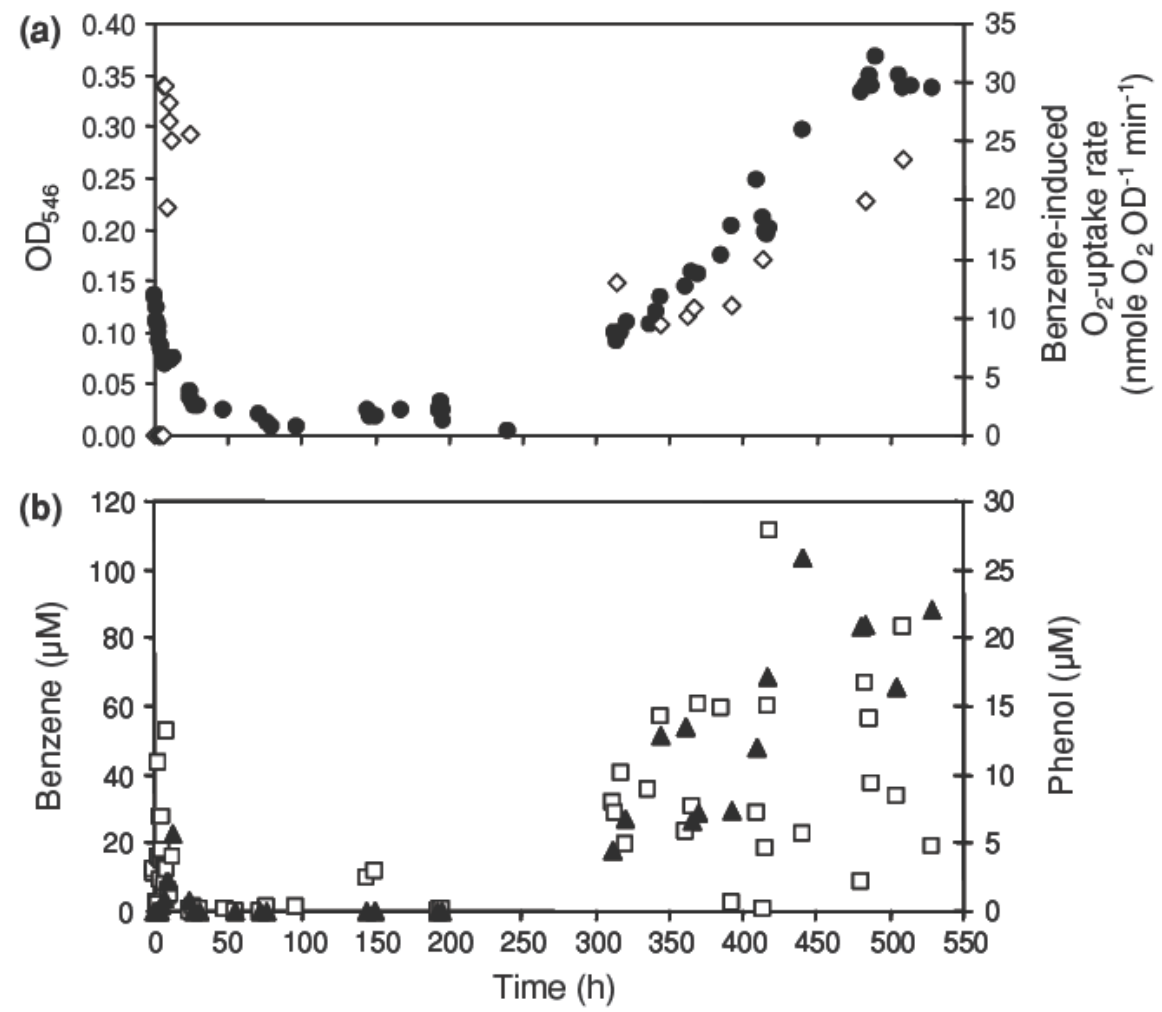

In the shift from succinate to benzene only (Fig. 4) the biomass stabilised at low $\mathrm{OD}_{546}$ values ranging between 0.01 and 0.03 after the second phase of wash-out. Not until about $300 \mathrm{~h}$ after the shift, growth resumed and eventually $\mathrm{OD}_{546}$ values around 0.34 were reached. Based on the biomass yields observed during batch growth with benzene, these biomass concentrations corresponded to the utilisation of about $1.2 \mathrm{mM}$ benzene, while the $\mathrm{OD}_{546}$ value of about 0.02 observed during the phase of low biomass can be explained by the consumption of $60 \mu \mathrm{M}$ benzene. According to calculations and measurements in a sterile control reactor, the latter is the benzene concentration establishing in the bioreactor under steady-state conditions in the absence of bacteria.

Apparently, the presence of succinate in the medium after the shift reduced the duration of the phase of low biomass concentrations. In the absence of succinate this phase lasted roughly $200 \mathrm{~h}$ in contrast to about $30 \mathrm{~h}$ when succinate was present. Interestingly, the second growth phase was characterised by fluctuations in the biomass concentration, which were accompanied by oscillations in the residual benzene concentrations (Figs. 3, 4). Possibly, the history of the culture which had gone through a first adaptation process only a few hours after the shift provoked a second growth phase, which again exhibited fluctuations but not as dramatic in the outcome as during the first induction phase. A similar positive effect of culture history has been observed for transitions of $E$. coli from glucose to a mixture of glucose plus 3-ppa (Kovarova et al. 1997). Shifting a culture a second time from glucose to the mixture after a period of growth with glucose only resulted in a two- to three-times shorter adaptation phase until steady-state was achieved.

In the two other transition experiments (where the inflowing succinate concentration was reduced to 0.35 and $0.125 \mathrm{mM}$ ) the bioreactors were run only up to around $150 \mathrm{~h}$ after the medium change. In this time period, no second increase of the biomass concentration was observed (data not shown). To test the state of the cells in the phase of such low biomass concentrations, in one experiment the medium composition was changed a second time increasing the succinate concentration from 0.35 to $1.25 \mathrm{mM}$. This 
change resulted in an immediate increase in biomass without transient accumulation of succinate in the culture, indicating that the cells were still active and that exposure to benzene did not inhibit biomass formation.

These observations led us to the assumption that in phases of low biomass concentrations the microbial activity was not high enough to provoke increased rates of diffusion of benzene from the gaseous into the liquid phase. To test this hypothesis the loss of benzene via the exhaust air was analysed for the transition from succinate to $1.25 \mathrm{mM}$ succinate plus $2 \mathrm{mM}$ benzene during the phase of low biomass (i.e. between 53 and $56 \mathrm{~h}$ after the shift). Surprisingly, the loss via the air ranged only around $3 \%$ of the incoming benzene. However, calculations as well as measurements during a sterile chemostat run suggested that benzene loss via air should be bigger than $75 \%$ in case of no consumption by the bacteria. Together with the low benzene and DOC concentrations in this phase (see below), this indicates that the incoming benzene was metabolised but not used for biomass production.

One might speculate, that in strain PKO1 the induction of adaptation mechanisms to increase tolerance towards the toxic aromatic compound benzene resulted in a rearrangement of the cellular metabolism characterised by a low biomass but mainly energy production from benzene. In addition, energy metabolism can be assumed to be less efficient in the presence of benzene due to the uncoupling character of the compound and its disturbing effects on energy-transducing proteins. Accordingly, in the solvent-tolerant strain P. putida S12 it was shown that during cultivation with glucose the presence of toluene (no growth substrate of the strain) decreased the yield from glucose and increased the maintenance coefficient (Isken et al. 1999). Energy-consuming cellular responses to solvent stress comprise a number of possible effects including the rigidification of the cellular membrane by alteration of the phospholipid composition, alterations of the cellular surface to make the cells less permeable, formation of vesicles to remove solvents from the cell surface, or eventually energy-dependent efflux pumps to remove the compounds from the cells (Ramos et al. 2002). For strain PKO1 a decrease in phospholipid content and changes in phospholipid composition upon exposure to toluene have been described (Fang et al. 2000).
Concentrations of substrates and degradation intermediates in the cultures: possible explanations for the behaviour of the cultures?

In all transition experiments (Figs. 3, 4) similarly high residual benzene concentrations between 10 and $60 \mu \mathrm{M}$ were detected during the initial phase of $12 \mathrm{~h}$. During periods of low biomass concentrations also benzene concentrations were low (around $1 \mu \mathrm{M}$ and below) but they were elevated (in general fluctuating between 5 and $40 \mu \mathrm{M}$ ) when the biomass reached higher values again. The phenol concentrations in the cultivation liquid followed a similar pattern whereas catechol concentrations were always below the detection limit. Eventually, also 2-hydroxymuconatesemialdehyde concentrations followed the biomass concentration pattern but they were always below $5 \mu \mathrm{M}$. Consequently, though DOC concentration did not vary significantly, higher biomass concentrations were in general accompanied by elevated DOC concentrations (about $2035 \mathrm{mg} \mathrm{C}^{-1}$ ). In contrast, succinate concentrations remained always below the detection limit.

As it is known that high concentrations of aromatic solvents can induce substrate-derived inhibitory effects and the accumulation of toxic intermediates (Leveau et al. 1999; Bordel et al. 2007; Munoz et al. 2007), intoxication of $R$. pickettii $P K O 1$ by benzene or by its metabolites was considered as possible explanation for the observed pattern of transient biomass formation and wash-out after the medium shift. However, for benzene a toxicity threshold of $1.18 \mathrm{mM}$ was found in P. putida and benzene did not inhibit growth of $R$. pickettii PKO1 at benzene concentrations below $0.61 .3 \mathrm{mM}$ and (Alagappan and Cowan 2003; Bringmann and Kühn 1980). The residual benzene concentrations during the chemostat experiments were always clearly below these values. Moreover, the data does not indicate an accumulation of any metabolite to concentrations which might have resulted in an inhibition of growth. Even the maximum phenol concentrations measured $(4050 \mu \mathrm{M})$ were clearly below the toxicity threshold of $0.7 \mathrm{mM}$ as determined for P. putida (Bringmann and Kühn 1980). Nevertheless, cells might have been stressed by the aromatic compounds although the concentrations were below the threshold toxicity level. Physiological stress can lead to the loss of the bacterial capability to use the stress-causing compound as a growth substrate, either 
temporarily or permanently (Kim and Jaffé 2007). Yet, in our experiments cells apparently were active in phases of decreasing or low biomass concentrations and consumed succinate and benzene because no wash-in of neither compound was detected. Additionally, the fact that succinate never accumulated in the cultivation liquid rules out the possibility of catabolite repression as found in batch experiments.

Finally, the possible existence of a threshold concentration for benzene utilisation was considered to explain the unexpected behaviour of the cultures after the medium shifts. As benzene probably is the effector molecule for the induction of the $t b u$ regulon (Olsen et al. 1997), transcription might be downregulated below certain concentrations of benzene in the cultivation liquid. Indeed, the apparent effectorbinding affinity of many regulatory proteins responsive to aromatic compounds is rather high, i.e. in the micromolar range (Tropel and van der Meer 2004). Thus, it is in the same range as the residual benzene concentrations found during phases of low biomass concentrations. In the initial phase after the medium shifts benzene degradation was not fully induced resulting in comparably high residual substrate concentrations that sustained expression of $t b u$ pathway enzymes. When fully induced, residual benzene concentrations possibly fell below a threshold level, so that enzyme expression was turned down again. Likewise, fluctuation in the concentrations of a signal molecule was assumed to provoke the overshooting observed at the level of mRNA transcripts during changes of growth conditions (Leveau et al. 1999; Baumann et al. 1996). However, if this explanation was true one should expect continuous oscillations in biomass and benzene concentrations, which was not observed. Moreover, Park et al. (2001) who claimed a threshold concentration for activation of toluene degradation (proceeding via the same $t b u$ pathway) in strain PKO1 of about $0.65 \mu \mathrm{M}$ found also that cells once induced degraded toluene down to concentrations below $0.1 \mu \mathrm{M}$ without loosing the capacity to metabolise toluene. Only when decreasing the toluene concentration in the feed of the system cells reacted by reducing the overall capacity for toluene degradation. In our system, however, the concentration of benzene in the feed remained constant after the medium change and, thus, there is no reason why the cells lost the capability once gained to consume benzene and produce biomass from it.

\section{Concluding remarks}

Although our investigations of the regulation of benzene catabolism in $R$. pickettii $\mathrm{PKO} 1$ remained at the phenomenological level, they showed that under carbon-limiting conditions (as predominantly found in nature) the adaptation process to growth with benzene is very complex being characterised by a first but transient increase in biomass followed by wash-out of cells leading to a phase of low biomass concentrations. Finally, the culture recovered and growth resumed. Such a behaviour was repeatedly observed in several medium shift experiments differing in the ratio of benzene to alternative substrate in the medium supplied to the culture after the transition. The behaviour of the strain was unexpected and not predictable from the information on the genetics and molecular biology of regulation of the $t b u$ pathway. Currently, the reasons for the collapse in biomass formation after the first rise are unknown but intoxications of the cells by benzene itself or the accumulation of toxic metabolites can be ruled out. All together, this underlines the necessity of phenomenological studies in addition to genetic analysis to better understand the behaviour of bacteria under natural conditions.

Acknowledgments This work was performed within the scope of the EU project QLK3 CT 200000731 and financially supported by grant BBW Nr. 99.0821 from the Swiss Federal Office for Education and Science.

\section{References}

Alagappan G, Cowan R (2003) Substrate inhibition kinetics for toluene and benzene degrading pure cultures and a method for collection and analysis of respirometric data for strongly inhibited cultures. Biotechnol Bioeng 83:798 809. doi:10.1002/bit.10729

Bally M, Egli T (1996) Dynamics of substrate consumption and enzyme synthesis in Chelatobacter heintzii during growth in carbon limited continuous culture with different mixtures of glucose and nitrilotriacetate. Appl Environ Microbiol 62:133 140

Baumann B, Snozzi M, Zehnder AJB, van der Meer JR (1996) Dynamics of denitrification activity of Paracoccus deni trificans in continuous culture during aerobic anaerobic changes. J Bacteriol 178:4367 4374

Bordel S, Munoz R, Diaz LF, Villaverde S (2007) New insights on toluene biodegradation by Pseudomonas putida F1: influence of pollutant concentration and excreted metabo lites. Appl Microbiol Biotechnol 74:857 866. doi:10.1007/ s0025300607248 
Bringmann G, Kühn R (1980) Comparison of the toxicity thresholds of water pollutants to bacteria, algae, and protozoa in the cell multiplication inhibition test. Water Res 14:231 241. doi:10.1016/0043 1354(80)90093 7

Bruins MR, Kapil S, Oehme FW (2000) Pseudomonas pickettii: a common soil and aerobic groundwater bacteria with pathogenic and biodegradation properties. Ecotoxicol Environ Saf 47:105 111. doi:10.1006/eesa.2000.1951

Byrne AM, Olsen RH (1996) Cascade regulation of the tolu ene 3 monooxygenase operon (tbuA1UBVA2C) of Burkholderia pickettii $\mathrm{PKO1:}$ role of the tbuA1 promotor (PtbuA1) in the expression of its cognate activator TbuT. J Bacteriol 178:6327 6337

Byrne AM, Kukor JJ, Olsen RH (1995) Sequence analysis of the gene cluster encoding toluene 3 monooxygenase from Pseudomonas pickettii PKO1. Gene 154:65 70. doi: 10.1016/0378 1119(94)00844 I

del Castillo T, Ramos JL (2007) Simultaneous catabolite repression between glucose and toluene metabolism in Pseudomonas putida is channeled through different signal pathways. J Bacteriol 189:6602 6610. doi:10.1128/ JB.00679 07

Duetz WA, van Andel JG (1991) Stability of TOL plasmid pWW0 in Pseudomonas putida mt 2 under non selective conditions in continuous culture. J Gen Microbiol 137:1369 1374

Egli T (1995) The ecological and physiological significance of the growth of heterotrophic microorganisms with mixtures of substrates. In: Jones GN (ed) Advances in microbial ecology. Plenum, New York, pp 305386

Egli T (2002) Microbial degradation of pollutants at low concentrations and in the presence of alternative carbon substrates: emerging patterns. In: Agathos SN, Reineke W (eds) Biotechnology for the environment: strategy and fundamentals. Kluwer, Dordrecht, pp 131139

Egli T, Weilenmann HU, El Banna T, Auling G (1988) Gram negative, aerobic, nitrilotriacetate utilizing bacteria from wastewater and soil. Syst Appl Microbiol 10:297 305

Evans CGT, Herbert D, Tempest DW (1970) The continuous cultivation of microorganisms construction of a che mostat. In: Norris JR, Ribbons DW (eds) Methods in microbiology (2). Academic Press, London, pp 277327

Fang J, Barvelon MJ, Alvarez PJJ (2000) Phospholipid com positional changes of five pseudomonas archetypes grown with and without toluene. Appl Microbiol Biotechnol 54:382 389. doi:10.1007/s002530000389

Finette BA, Gibson DT (1988) Initial studies on the regulation of toluene degradation by Pseudomonas putida F1. Bio catalysis 2:29 37. doi:10.3109/10242428808998177

Gulensoy N, Alvarez PJ (1999) Diversity and correlation of specific aromatic hydrocarbon biodegradation capabili ties. Biodegradation 10:331 340. doi:10.1023/A:10083 18405882

Heiss G, Stolz A, Kuhm AE, Müller C, Klein J, Altenbuchner J, Knackmuss H J (1995) Characterization of a 2, 3 dihydroxybiphenyl dioxygenase from the naphtalenesulf onate degrading bacterium strain BN6. J Bacteriol 177:5865 5871

Isken S, Derks A, Wolffs PF, de Bont JA (1999) Effect of organic solvents on the yield of solvent tolerant Pseudo monas putida S12. Appl Environ Microbiol 65:2631 2635
Jindrova E, Chocova M, Demnerova K, Brenner V (2002) Bacterial aerobic degradation of benzene toluene, ethyl benzene and xylene. Folia Microbiol (Praha) 47:83 93. doi:10.1007/BF02817664

Johnson DR, Park J, Kukor JJ, Abriola LM (2006) Effect of carbon starvation on toluene degradation activity by tol uene monooxygenase expressing bacteria. Biodegradation 17:437 445. doi:10.1007/s10532005 $9014 \mathrm{x}$

Kahng H Y, Byrne AM, Olsen RH, Kukor JJ (2000) Charac terization and role of $t b u X$ in utilization of toluene by Ralstonia pickettii PKO1. J Bacteriol 182:1232 1242. doi:10.1128/JB.182.5.1232 1242.2000

Kim H, Jaffé PR (2007) Spatial distribution and physiological state of bacteria in a sand column experiment during the biodegradation of toluene. Water Res 41:2089 2100 . doi:10.1016/j.watres.2007.02.018

Kovarova K, Käch A, Chaloupka V, Egli T (1997) Cultivation of Escherichia coli with mixtures of 3 phenylpropionic acid and glucose: dynamics of growth and substrate consumption. Biodegradation 7:2619 2624

Kukor JJ, Olsen RH (1990) Molecular cloning, characteriza tion, and regulation of a Pseudomonas pickettii PKO1 gene encoding phenol hydroxylase and expression of the gene in Pseudomonas aeruginosa PAO1c. J Bacteriol 172:4624 4630

Kukor JJ, Olsen RH (1991) Genetic organization and regula tion of a meta cleavage pathway for catechols produced from catabolism of toluene, benzene, phenol, and cresols by Pseudomonas pickettii PKO1. J Bacteriol 173:4587 4594

Kukor JJ, Olsen RH (1992) Complete nucleotide sequence of $t b u D$, the gene encoding phenol/cresol hydroxylase from Pseudomonas pickettii PKO1, and functional analysis of the encoded enzyme. J Bacteriol 174:6518 6526

Kukor JJ, Olsen RH (1996) Catechol 2, 3 dioxygenases func tional in oxygen limited (hypoxic) environments. Appl Environ Microbiol 62:1728 1740

Leahy JG, Olsen RH (1997) Kinetics of toluene degradation by toluene oxidizing bacteria as a function of oxygen con centration, and the effect of nitrate. FEMS Microbiol Ecol 23:23 30. doi:10.1111/j.1574 6941.1997.tb00387.x

Leddy MB, Phipps DW, Ridgway HF (1995) Catabolite med iated mutations in alternate toluene degradative pathways in Pseudomonas putida. J Bacteriol 177:4713 4720

Leveau JHJ, König F, Füchslin HP, Werlen C, van der Meer JR (1999) Dynamics of multigene expression during cata bolic adaptation of Ralstonia eutropha JMP134 (pJP4) to the herbicide 2, 4 dichlorophenoxyacetate. Mol Microbiol 33:396 406. doi:10.1046/j.1365 2958.1999.01483.x

Lovley DR (2000) Anaerobic benzene degradation. Biodegra dation 11:107 116. doi:10.1023/A:1011191220463

Massol Deya A, Weller R, Rios Hernandez L, Zhou JZ, Hickey RF, Tiedje JM (1997) Succession and conver gence of biofilm communities in fixed film reactors treating aromatic hydrocarbons in groundwater. Appl Environ Microbiol 63:270 276

Morita RY (1993) Bioavailability of energy and the starvation state. In: Kjelleberg S (ed) Starvation in bacteria. Plenum, New York, pp 123

Munoz R, Diaz LF, Bordel S, Villaverde S (2007) Inhibitory effects of catechol accumulation on benzene biodegradation 
in Pseudomonas putida F1 cultures. Chemosphere 68: 244 252. doi:10.1016/j.chemosphere.2007.01.016

Olsen RH, Kukor JJ, Kaphammer B (1994) A novel toluene 3 monooxygenase pathway cloned from Pseudomonas pickettii PKO1. J Bacteriol 176:3749 3756

Olsen RH, Mikesell MD, Kukor JJ, Byrne AM (1995) Physio logical attributes of microbial BTEX degradation in oxygen limited environments. Environ Health Perspect 103(Suppl 5):49 51. doi:10.2307/3432479

Olsen RH, Kukor JJ, Byrne AM, Johnson GR (1997) Evidence for the evolution of a single component phenol/cresol hydroxylase from a multicomponent toluene monooxy genase. J Ind Microbiol Biotechnol 19:360 368. doi: 10.1038/sj.jim.2900453

Park J, Chen YM, Kukor JJ, Abriola LM (2001) Influence of substrate exposure history on biodegradation in a porous medium. J Contam Hydrol 51:233 256. doi:10.1016/ S0169 7722(01)00125 5

Park J, Malinverni J, Adriaens P, Kukor JJ (2003) Quantitative structure activity relationship (QSAR) analysis of aromatic effector specificity in NtrC like transcriptional activators from aromatic oxidizing bacteria. FEMS Microbiol Lett 224:45 52. doi:10.1016/S0378 1097(03)00400 2

Ramos JL, Duque E, Gallegos MT, Godoy P, Ramos Gonzalez MI, Rojas A, Teran W, Segura A (2002) Mechanisms of solvent tolerance in Gram negative bacteria. Annu Rev Microbiol 56:743 768. doi:10.1146/annurev.micro.56.012 302.161038

Rüegg I, Hafner T, Bucheli Witschel M, Egli T (2007) Dynamics of benzene and toluene degradation in Pseu domonas putida $\mathrm{F} 1$ in the presence of the alternative substrate succinate. Eng Life Sci 7:331 342. doi:10.1002/ elsc. 200720202

Shingler V (2003) Integrated regulation in response to aromatic compounds: from signal sensing to attractive behaviour. Environ Microbiol 5:1226 1241. doi:10.1111/j.1462 2920.2003.00472.x
Sigg L, Stumm W (1996) Eine Einführung in die Chemie wässriger Lösungen und natürlicher Gewässer. Hoch schulverlag an der ETH Zürich, Zürich

Smith MR (1994) The physiology of aromatic hydrocarbon degrading bacteria. In: Ratledge $\mathrm{C}$ (ed) Biochemistry of microbial degradation. Kluwer, Dordrecht, pp 347378

Spormann AM, Widdel F (2000) Metabolism of alkylbenzenes, alkanes and other hydrocarbons in anaerobic bacteria. Bio degradation 11:85 105. doi:10.1023/A:1011122631799

Stumm W, Morgan JJ (1981) Aquatic chemistry: an introduc tion emphasizing chemical equilibria in natural waters. Wiley, New York

Tao Y, Fishman A, Bentley WE, Wood TK (2004) Oxidation of benzene to phenol, catechol, and 1,2,3 trihydroxy benzene by toluene 4 monooxygenase of Pseudomonas mendocina KR1 and toluene 3 monooxygenase of Rals tonia pickettii PKO1. Appl Environ Microbiol 70:3814 3820. doi:10.1128/AEM.70.7.3814 3820.2004

Tropel D, van der Meer JR (2004) Bacterial transcriptional regulators for degradation pathways of aromatic com pounds. Microbiol Mol Biol Rev 68:474 500. doi:10.1128/MMBR.68.3.474 500.2004

Vroblesky DA, Chapelle FH (1994) Temporal and spatial changes of terminal electron accepting processes in a petroleum hydrocarbon contaminated aquifer and the significance for contaminant biodegradation. Water Resour Res 30:1561 1570. doi:10.1029/94WR00067

Yerushalmi L, Manuel MF, Guiot SR (1999) Biodegradation of gasoline and BTEX in a microaerophilic biobarrier. Bio degradation 10:341 352. doi:10.1023/A:1008327815105

Yu H, Kim BJ, Rittmann BE (2001) The roles of intermediates in biodegradation of benzene, toluene and $p$ xylene by Pseudomonas putida F1. Biodegradation 12:455 463. doi:10.1023/A:1015008627732 